\title{
Signaling pathways in melanoma biology and new targeted therapeutic approaches
}

\author{
Corresponding author: \\ Aleksander Kiełbik, Faculty \\ of Medicine, Wroclaw Medical \\ University, J. Mikulicza-Radeckiego \\ Str. 5, 50-345 Wroclaw, Poland \\ e-mail: akielbik6@gmail.com
}

Medical Research Journal 2019; Volume 4, Number 3, 184-188 10.5603/MRJ.a2019.0033 Copyright (C) 2019 Via Medica ISSN 2451-2591

\begin{abstract}
Despite the broad prevention programs and early detection and therapy progress, melanoma of skin is still responsible for $0.6 \%$ of deaths caused by tumour disease. Every year 300,000 patients are diagnosed and 60,000 die from the most malignant of skin cancer. Generally, melanoma is formed as a result of mutation of growth pathways responsible for proliferation and apoptosis. One of the most investigated pathway, mutated in $90 \%$ of melanomas, is RAS $>$ RAF $>M E K>E R K$ also known as mitogen-activated protein kinase (MAPK) pathway. The second one is phosphoinositide-3-OH kinase (PI3K) pathway. The better understanding of melanoma biology resulted in research of inhibitors, which can affect presented pathways and prevent uncontrolled proliferation of melanoma. The BRAF inhibitors vemurafenib and dabrafenib and MEK inhibitor trametinib seem to be the most successful ones. Recent advances in biology of melanoma provided new interesting therapeutic targets. One of the most inquiring is microphthalmia associated transcription factor (MITF), the principal regulator of melanocyte lineage. MITF perform the role of so-called 'survival' or 'addiction' oncogene. Moreover, the interleukin-1 receptor-associated kinases (IRAKs) might clarify the connection between the inflammatory environment and melanoma carcinogenesis. IRAKs play a key role as mediators of toll-like receptor (TLR) and interleukin-1 receptor (IL1R) in inflammation signalling processes. Moreover, it was observed that metformin cause cell cycle arrest in melanoma cells, secondly leading to activation of autophagy and apoptosis. Although due to targeted and immunotherapy the prognosis of patients with metastatic melanoma is incomparably better, melanoma in its advanced stadium is still predominantly lethal. Therefore, the most present research concentrates on acquired resistance against targeted therapy.

Key words: melanoma, targeted therapy, dermatology, oncology
\end{abstract}

Med Res J 2019; 4 (3): 184-188

\section{Introduction}

Melanocytes are melanin producing cells that anatomically exist mainly in the epidermal cell population, but have origins in neural crest cells. As a pigment producer they are responsible for skin and hair colour. They also play a key role in prevention of skin damage caused by UV radiation. In unaffected skin they are situated in basal layer of epidermis where their metabolism is regulated by keratinocytes [1]. Mutations in oncogenes enable melanocytes to avoid this regulation, then unrestricted spread and consequently develop the skin cancer, melanoma. Undergoing radial and vertical growth phase melanoma can form malignant tumour and then infiltrate the vascular or lymphatic system [2]. Considering different clinical pathology diagnosis, etiology, biologic properties, and prognosis among cutaneous melanoma most authors distinguish nodular melanoma (NM), superficial spreading melanoma (SSM), lentigo maligna melanoma (LMM), and acrallentiginous melanoma (ALM) [3]. Studies on cell-signaling mechanisms enabled to determine the pathways controlling growth and division of cells. Some of them become often hyperactivated leading to uncontrolled proliferation of cancer cells.

\section{Epidemiology}

As the epidemiological research shows that melanoma of skin is responsible for $0.6 \%$ of deaths caused by tumour disease. Every year 300,000 patients are diag- 
nosed and 60,000 die from the most malignant of skin cancer [4]. Normally, once the skin cancers are early diagnosed and receive appropriate management, they reach $95 \%$ of 5 -year survival rate. However, cutaneous malignant melanoma shows relevant higher morbidity and mortality and is responsible for $65 \%$ of all skin cancer deaths [5]. There are several factors, which contribute to the increasing incidence of skin cancers. Among them, the exposure to sunlight and other sources of UV irradiation is often associated with development of melanoma. Moreover, rising exploitation of sunlamps, tanning beds and the depletion of the earth's ozone result in higher exposure to radiation and secondly in exacerbation of the skin cancers incidents [6].

\section{Melanoma biology}

Melanoma occurs as a result of multiple mutations that cause deregulation of several principal cell signalling pathways, which leads to uncontrolled proliferation and formation of melanoma. Understanding these processes helps to undertake specific and personalized therapies.

\section{The role of MAPK, PI3K and PTEN pathways}

One of the most investigated pathway, mutated in $90 \%$ of melanomas, is RAS $>$ RAF $>$ MEK $>$ ERK (also known as mitogen-activated protein kinase (MAPK) pathway). Its activation triggers the mitosis of cells resulting in uncontrolled proliferation of cells [7]. In $50-70 \%$ of cases, the pathway is upregulated by BRAF mutation (the most common is V600EBRAF) and in $15-30 \%$ by $N$-ras mutation $[8,9]$.

It was proven that the single mutation might have an effect on several aspects of tumour genesis. In melanoma case the BRAF mutation, besides increased proliferation of melanoma cells results in upregulation of VEGF, the angiogenic factor [10].

The second major melanoma growth pathway is phosphoinositide-3-OH kinase (PI3K) pathway. PI3Ks are a family of lipid kinases acting as lipid second messengers that affect downstream regulators, among others protein kinase $B(P K B)$. PI3K signalling regulates cell survival, proliferation and growth. The supervisory function in this pathway is performed by PTEN, the factor responsible for signal termination. PTEN loss of function, resulting in uncontrolled cancer proliferation, is present in 5-20\% of melanoma [11].

Significant is that NRAS mutation does not generally occur simultaneously neither with BRAF nor with PTEN mutation, whereas the changes in both BRAF and PTEN are present in $20 \%$ of cases. This observation supports the conclusion that BRAF and PI3K are activated downstream of RAS. BRAF and PTEN operate

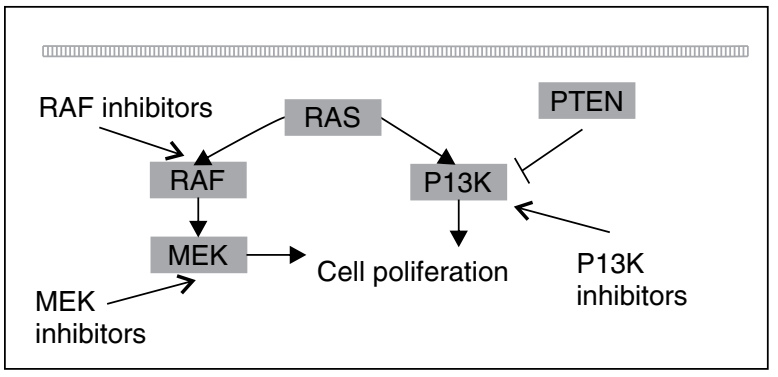

Figure 1. Cell proliferation pathways in melanoma and possibilities of its targeted inhibition [15]. Main pathways involved in melanoma cell survival, proliferation, growth RAS/RAF/MEK pathway and PI3K pathway are presented as an intracellular processes together which possibilities of their inhibition

on distinct genetic pathways and could cooperate to promote melanoma proliferation [12, 13]. Moreover, some of the $\mathrm{N}$-ras mutations seems to be typical for congenital melanocytic neavi, but are not characteristic to dysplastic melanocytic neavi [14].

\section{The role of MITF and $\mathrm{p} 16$ regulators}

An interesting regulator of melanocyte lineage is microphthalmia associated transcription factor (MITF). MITF perform the role of so-called 'survival' or 'addiction' oncogenes [16]. It was observed that MITF is essential for cell proliferation and survival, but its level must be strictly controlled. Increased MITF leads to cell cycle arrest, whereas too low level or complete absence may result in cell apoptosis. Moreover it was noticed that MITF can corporate with ${ }^{\mathrm{V} 600 \mathrm{E}} \mathrm{BRAF}$ and act as an additive oncogene [16, 17].

Melanoma occurs not always as the result of spontaneous mutations. Germline changes in the $\mathrm{p} 16^{\mathrm{IN}}$ K4a gene have been identified in approximately half of the families with hereditary melanoma [18]. Bennett et al. observed that mutations in p16 result in extremely increased lifetime risk of melanoma [19]. In normal physiological state $\mathrm{p} 16^{\text {ink4a }}$ enables call to enter the senescence stage. When this function is lost, cells gain the ability of proliferation [20]. Upregulation of p16ink4a can also induce the formation benign naevi [15].

\section{The role of IRAK in deregulation of inflammation}

There seems to be relevant correlation between inflammation and genesis of tumours. Some of the pathways partly clarify this reciprocal connection. The interleukin-1 receptor-associated kinases (IRAKs) play a key role as mediators of toll-like receptor (TLR) and interleukin-1 receptor (IL1R) signalling processes. It was revealed that TLR/IL1R-mediated signalling con- 


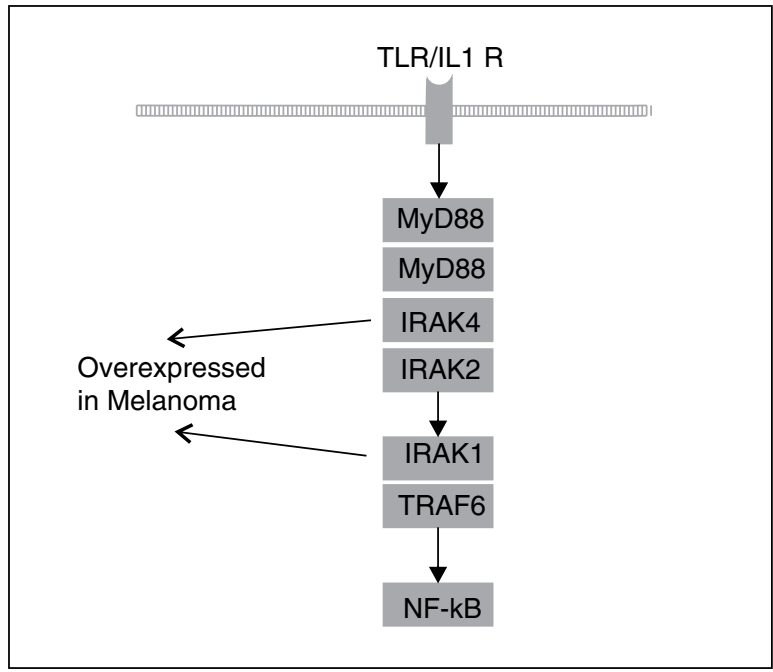

Figure 2. Immune signalling via IRAK family network and its role in inflammation process [21, 23]. The interleukin-1 receptor-associated kinases (IRAKs) play a key role as mediators of toll-like receptor (TLR) and interleukin-1 receptor (IL1R) presented on the cell membrane. The Intracellular signalling processes results in increasing NF- $\kappa$ B activity and deregulation of inflammation. Two kinases IRAK1 and IRAK4 are overexpressed in melanoma cell lines and their role is currently investigated

trols diverse cellular processes including inflammation, apoptosis, and cellular differentiation [21, 22]. Importance of IRAK receptors in deregulation of inflammation, increasing NF- $\kappa \mathrm{B}$ activity and formation of cancer is currently investigated.

IRAK1 and IRAK4 are overexpressed and activated in melanoma cell lines. The attenuating IRAK-1,-4 signalling with pharmacological inhibitors in vitro increased vinblastine impact on melanoma cell death [23]. Further, in a xenograft mouse, combined therapy with IRAK inhibitors had significant impact on prolonged survival [23]. IRAKs seem to be promising targets to enhance the therapeutic response, but until now there are no studies proving the effectiveness of IRAK inhibitors on melanoma patients in vivo.

\section{Targeted treatment possibilities}

The better understanding of melanoma biology resulted in the research of inhibitors, which can affect presented pathways preventing uncontrolled proliferation of melanoma. The most successful seem to be BRAF inhibitors vemurafenib [24] and dabrafenib [25] and MEK inhibitor trametinib [26]. The trials on vemurafenib showed the response rates of more than $50 \%$ in patients with metastatic melanoma with the V600EBRAF mutation. Moreover it significantly reduced the risk of death and disease progression, as compared with chemotherapy with dacarbazine [27]. Letter on, the studies on dabrafenib showed similar results on patient with advanced-stage metastatic melanoma [25]. As the responses seem to be short-lived next trials were focused on MEK inhibition with trametinib [28].

Long-term investigation showed that patients undergoing targeted therapy developed resistance to BRAF inhibitors. MAPK reactivation mechanisms were acquired by $N$-ras mutations. Mutant BRAF amplification and alternative splicing were detected in $70 \%$ of patients. PI3K-PTEN-AKT-upregulation was observed in $22 \%$ of progressive melanoma disease [29]. Moreover, the most severe side effect of BRAF inhibition was the development of secondary skin tumours, originating from a paradoxical activation of the MAPK pathway in cells without $B R A F$ mutation [27]. The potential solution to this problem may be provided by combined therapy. The results of trial with dabrafenib plus trametinib vs vemurafenib monotherapy showed encouraging results enhancing overall survival in previously untreated patients with metastatic melanoma [30-32]. Targeted medicaments currently approved by FDA for metastatic melanoma targeted treatment ware presented in Table 1.

Although presented results seem to be promising there is still the vast part of responses that are not typically complete or durable. Therefore, the major focus is required to identify and target the mechanisms of resistance. Not only combined approaches among targeted but also immune therapy might provide the extended overall survival and enhance the quality of life of the patients with metastatic melanoma.

Table 1. Targeted medicaments currently approved by FDA for metastatic melanoma targeted treatment

\begin{tabular}{lcc}
\hline Name of medicaments & Pathway & Studies \\
\hline Encorafenib and binimetinib combination & BRAF MEK kinases inhibitor & {$[39]$} \\
Cobimetinib and vemurafenib combination & BRAF and MEK kinases inhibitor & {$[40]$} \\
Trametinib & MEK inhibitor & {$[28]$} \\
Trametinib and dabrafenib combination & BRAF and MEK kinases inhibitor & {$[30-32]$} \\
Dabrafenib & BRAF kinase inhibitor & {$[25]$} \\
Vemurafenib & BRAF kinase inhibitor & {$[41]$} \\
\hline
\end{tabular}




\section{Metformin induces AMPK-dependent and} -independent melanoma cell death

Metformin is generally used for Diabetes treatment but it might find its application in oncology. The mechanism of action of metformin has not been fully elucidated. So far, the activation of AMP-activated protein kinase (AMPK) in mitochondria seems to provide a rational explanation [33].

Metformin uses AMPK-dependent and -independent pathway to induce melanoma cell death [34]. It was also observed that metformin cause cell cycle arrest in melanoma cells, secondly leading to activation of autophagy and apoptosis [35]. Moreover, Hirsch et al. have proven that Metformin inhibits the inflammatory response preventing cellular transformation and inhibiting cancer growth [36].

Metformin is known to alternate the mitochondrial metabolism, what secondly influence the tumour microenvironment [37]. Its role as an adjuvant medicament against melanoma is currently undergoing trials among others with targeted therapy agents [34, 38].

\section{Conclusion}

Years of research on melanoma cell biology finally gave rise to relatively safe and efficient therapies. The knowledge of specific proliferation pathways together with progress in oncoimmunology resulted in the development of targeted drugs. Those can be applied, when other therapeutic options, such as surgery are exploited. Although the prognosis of patients with metastatic melanoma is incomparably better, melanoma in its advanced stadium is still predominantly lethal. Therefore, the most present research concentrates on acquired resistance against targeted therapy.

\section{Acknowledgements}

The work was created as part of the activity of the Student Research Group of Biology of Cancer Cell at the Wroclaw Medical University (SKN No. 148) and partially by Statutory Funds of Wroclaw Medical University (Department of Molecular and Cellular Biology).

\section{References}

1. Cichorek M, Wachulska M, Stasiewicz A, et al. Skin melanocytes: biology and development. Postepy Dermatol Alergol. 2013; 30(1) 30-41, doi: 10.5114/pdia.2013.33376, indexed in Pubmed: 24278043

2. Clark WH, Elder DE, Guerry D, et al. Model predicting survival in stage I melanoma based on tumor progression. J Natl Cancer Inst. 1989; 81(24): 1893-1904, doi: 10.1093/jnci/81.24.1893, indexed in Pubmed: 2593166
3. Scolyer RA, Long GV, Thompson JF. Evolving concepts in melanoma classification and their relevance to multidisciplinary melanoma patient care. Mol Oncol. 2011; 5(2): 124-136, doi: 10.1016/j.molonc.2011.03.002, indexed in Pubmed: 21482206.

4. Bray F, Ferlay J, Soerjomataram I, et al. Global cancer statistics 2018: GLOBOCAN estimates of incidence and mortality worldwide for 36 cancers in 185 countries. CA Cancer J Clin. 2018; 68(6): 394-424, doi: 10.3322/caac.21492, indexed in Pubmed: 30207593.

5. Cummins DL, Cummins JM, Pantle H, et al. Cutaneous malignant melanoma. Mayo Clin Proc. 2006; 81(4): 500-507, doi: 10.4065/81.4.500, indexed in Pubmed: 16610570.

6. Gallagher RP, Spinelli JJ, Lee TK. Tanning beds, sunlamps, and risk of cutaneous malignant melanoma. Cancer Epidemiol Biomarkers Prev. 2005; 14(3): 562-566, doi: 10.1158/1055-9965.EPI-04-0564, indexed in Pubmed: 15767329

7. Wellbrock C, Arozarena I. The Complexity of the ERK/MAP-Kinase Pathway and the Treatment of Melanoma Skin Cancer. Front Cell Dev Biol. 2016; 4: 33, doi: 10.3389/fcell.2016.00033, indexed in Pubmed: 27200346

8. Kwong LN, Costello JC, Liu H, et al. Oncogenic NRAS signaling differentially regulates survival and proliferation in melanoma. Nat Med. 2012; 18(10): 1503-1510, doi: 10.1038/nm.2941, indexed in Pubmed: 22983396.

9. Davies $\mathrm{H}$, Bignell GR, Cox $\mathrm{C}$, et al. Mutations of the BRAF gene in human cancer. Nature. 2002; 417(6892): 949-954, doi: 10.1038/nature00766, indexed in Pubmed: 12068308.

10. Sharma A, Trivedi NR, Zimmerman MA et al. Mutant V599EB-Raf regulates growth and vascular development of malignant melanoma tumors. Cancer Res. 2005; 65(6): 2412-2421, doi: 10.1158/0008-5472. CAN-04-2423, indexed in Pubmed: 15781657

11. Wu H, Goel V, Haluska FG. PTEN signaling pathways in melanoma Oncogene. 2003; 22(20): 3113-3122, doi: 10.1038/sj.onc.1206451, indexed in Pubmed: 12789288.

12. Tsao $H$, Goel $V, W u H$, et al. Genetic interaction between NRAS and BRAF mutations and PTEN/MMAC1 inactivation in melanoma. J Invest Dermatol. 2004; 122(2): 337-341, doi: 10.1046/j.0022202X.2004.22243.x, indexed in Pubmed: 15009714.

13. Rodriguez-Viciana P, Marte BM, Warne PH, et al. Phosphatidylinositol 3' kinase: one of the effectors of Ras. Philos Trans R Soc Lond B Biol Sci. 1996; 351(1336): 225-31; discussion 231, doi: 10.1098/rstb.1996.0020, indexed in Pubmed: 8650270.

14. Papp T, Pemsel H, Rollwitz I, et al. Mutational analysis of N-ras, p53 CDKN2A (p16(INK4a)), p14(ARF), CDK4, and MC1R genes in human dysplastic melanocytic naevi. J Med Genet. 2003; 40(2): E14, doi: 10.1136/jmg.40.2.e14, indexed in Pubmed: 12566532.

15. Gray-Schopfer V, Wellbrock $C$, Marais R. Melanoma biology and new targeted therapy. Nature. 2007; 445(7130): 851-857, doi: 10.1038/nature05661, indexed in Pubmed: 17314971

16. Garraway LA, Widlund HR, Rubin MA, et al. Integrative genomic analyses identify MITF as a lineage survival oncogene amplified in malignant melanoma. Nature. 2005; 436(7047): 117-122, doi: 10.1038/nature03664, indexed in Pubmed: 16001072.

17. Levy C, Khaled M, Fisher DE. MITF: master regulator of melanocyte development and melanoma oncogene. Trends Mol Med. 2006; 12(9): 406-414, doi: 10.1016/j.molmed.2006.07.008, indexed in Pubmed: 16899407.

18. Zuo L, Weger J, Yang Q, et al. Germline mutations in the p16INK4a binding domain of CDK4 in familial melanoma. Nat Genet. 1996; 12(1): 97-99, doi: 10.1038/ng0196-97, indexed in Pubmed: 8528263

19. Bennett DC Human melanocyte senescence and melanoma susceptibility genes. Oncogene. 2003; 22(20): 3063-3069, doi: 10.1038/sj.onc.1206446, indexed in Pubmed: 12789281.

20. Alcorta DA, Xiong Y, Phelps D, et al. Involvement of the cyclin-dependent kinase inhibitor p16 (INK4a) in replicative senescence of normal human fibroblasts. Proc Natl Acad Sci U S A. 1996; 93(24): 13742-13747, doi: 10.1073/pnas.93.24.13742, indexed in Pubmed: 8943005.

21. Rhyasen GW, Starczynowski DT. IRAK signalling in cancer. $\mathrm{Br} \mathrm{J}$ Cancer. 2015; 112(2): 232-237, doi: 10.1038/bjc.2014.513, indexed in Pubmed: 25290089.

22. Lin KM, Hu W, Troutman TyD, et al. IRAK-1 bypasses priming and directly links TLRs to rapid NLRP3 inflammasome activation. Proc Natl Acad Sci U S A. 2014; 111(2): 775-780, doi: 10.1073/pnas.1320294111, indexed in Pubmed: 24379360

23. Srivastava R, Geng D, Liu Y, et al. Augmentation of therapeutic responses in melanoma by inhibition of IRAK-1,-4. Cancer Res. 2012 72(23): 6209-6216, doi: 10.1158/0008-5472.CAN-12-0337, indexed in Pubmed: 23041547 
24. Bollag G, Hirth P, Tsai J, et al. Clinical efficacy of a RAF inhibitor needs broad target blockade in BRAF-mutant melanoma. Nature. 2010; 467(7315): 596-599, doi: 10.1038/nature09454

25. Hauschild A, Grob JJ, Demidov L, et al. Dabrafenib in BRAF-mutated metastatic melanoma: a multicentre, open-label, phase 3 randomised controlled trial. The Lancet. 2012; 380(9839): 358-365, doi: 10.1016/s0140-6736(12)60868-x.

26. Gilmartin AG, Bleam MR Groy A et al. GSK1120212 (JTP-74057) is an inhibitor of MEK activity and activation with favorable pharmacokinetic properties for sustained in vivo pathway inhibition. Clin Cancer Res. 2011; 17(5): 989-1000, doi: 10.1158/1078-0432.CCR-10-2200, indexed in Pubmed: 21245089

27. Chapman PB, Hauschild A, Robert C, et al. BRIM-3 Study Group. Improved survival with vemurafenib in melanoma with BRAF V600E mutation. N Engl J Med. 2011; 364(26): 2507-2516, doi: 10.1056/NEJ Moa1103782, indexed in Pubmed: 21639808.

28. Flaherty KT, Robert C, Hersey P, et al. METRIC Study Group. Improved survival with MEK inhibition in BRAF-mutated melanoma. N Engl Med. 2012; 367(2): 107-114, doi: 10.1056/NEJMoa1203421, indexed in Pubmed: 22663011.

29. Shi $\mathrm{H}$, Hugo W, Kong $X$, et al. Acquired resistance and clonal evolution in melanoma during BRAF inhibitor therapy. Cancer Discov. 2014; 4(1): 80-93, doi: 10.1158/2159-8290.CD-13-0642, indexed in Pubmed: 24265155.

30. Robert C, Karaszewska B, Schachter J, et al. Improved overall survival in melanoma with combined dabrafenib and trametinib. $N$ Engl $J$ Med. 2015; 372(1): 30-39, doi: 10.1056/NEJMoa1412690, indexed in Pubmed: 25399551.

31. Long GV, Eroglu Z, Infante J, et al. Long-Term Outcomes in Patients With BRAF V600-Mutant Metastatic Melanoma Who Received Dabrafenib Combined With Trametinib. J Clin Oncol. 2018; 36(7): 667-673, doi: 10.1200/JCO.2017.74.1025, indexed in Pubmed: 28991513

32. Long G, Stroyakovskiy D, Gogas H, et al. Dabrafenib and trametinib versus dabrafenib and placebo for Val600 BRAF-mutant melanoma: a multicentre, double-blind, phase 3 randomised controlled trial. The Lancet 2015; 386(9992): 444-451, doi: 10.1016/s0140-6736(15)60898-4
33. Zhou G, Myers R, Li Y, et al. Role of AMP-activated protein kinase in mechanism of metformin action. J Clin Invest. 2001; 108(8): 1167-1174, doi: 10.1172/JCl13505, indexed in Pubmed: 11602624

34. Jaune E, Rocchi S. Metformin: Focus on Melanoma. Frontiers in Endocrinology. 2018; 9, doi: 10.3389/fendo.2018.00472.

35. Tomic T, Botton $\mathrm{T}$, Cerezo M, et al. Metformin inhibits melanoma development through autophagy and apoptosis mechanisms. Cell Death Dis. 2011; 2: e199, doi: 10.1038/cddis.2011.86, indexed in Pubmed: 21881601

36. Hirsch HA, lliopoulos D, Struhl K. Metformin inhibits the inflammatory response associated with cellular transformation and cancer stem cell growth. Proc Natl Acad Sci U S A. 2013; 110(3): 972-977, doi: 10.1073/pnas.1221055110, indexed in Pubmed: 23277563.

37. Liu X Romero IL, Litchfield LM et al Metformin Targets Central Carbon Metabolism and Reveals Mitochondrial Requirements in Human Cancers. Cell Metab. 2016; 24(5): 728-739, doi: 10.1016/j. cmet.2016.09.005, indexed in Pubmed: 27746051.

38. Niehr $F$, von Euw $E$, Attar N, et al. Combination therapy with vemurafenib (PLX4032/RG7204) and metformin in melanoma cell lines with distinct driver mutations. J Transl Med. 2011; 9: 76, doi: 10.1186/14795876-9-76, indexed in Pubmed: 21609436.

39. Dummer R, Ascierto P, Gogas $\mathrm{H}$, et al. Overall survival in patients with BRAF-mutant melanoma receiving encorafenib plus binimetinib versus vemurafenib or encorafenib (COLUMBUS): a multicentre, open-label, randomised, phase 3 trial. The Lancet Oncology. 2018; 19(10): 1315-1327, doi: 10.1016/s1470-2045(18)30497-2

40. Ascierto P McArthur G Dréno B et al Cobimetinib combined with vemurafenib in advanced BRAFV600-mutant melanoma (coBRIM): updated efficacy results from a randomised, double-blind, phase 3 trial. The Lancet Oncology. 2016; 17(9): 1248-1260, doi: 10.1016/s1470-2045(16)30122-x

41. Maio M, Lewis K, Demidov L, et al. Adjuvant vemurafenib in resected, BRAF V600 mutation-positive melanoma (BRIM8): a randomised, double-blind, placebo-controlled, multicentre, phase 3 trial. The Lancet Oncology. 2018; 19(4): 510-520, doi: 10.1016/s14702045(18)30106-2 